\title{
Literaturbericht
}

\author{
Andreas Busen und Thorsten Thiel
}

\section{Freiheit, Gerechtigkeit und Demokratie: Renovierungsarbeiten am Neo-Republikanismus}

Innerhalb der vergangenen zwanzig Jahre hat sich innerhalb der zeitgenössischen Politischen Theorie eine Position entwickelt, die sich selbst den Stellenwert einer Alternative zum als hegemonial empfundenen Liberalismus zuschreibt: der NeoRepublikanismus. Zentral für die Entwicklung dieser Theorie sind die Arbeiten von Quentin Skinner und Philip Pettit. Skinner ist es, der den ideengeschichtlichen Anschub für das Argument formuliert hat. Sein Werk zeigt, wie sich die republikanische Idee in der römischen Republik und dann in der ersten Blütephase der italienischen Stadtstaaten der Renaissance formierte, um schließlich von Autoren wie James Harrington, John Milton oder Algernon Sidney im England des 17. Jahrhundert als Gegenentwurf zu liberalen Staatstheorien à la Hobbes aktualisiert zu werden. Es zeigt aber auch, dass diese Aktualisierung nicht nachhaltig wirkte. Vielmehr sei der Republikanismusbegriff ab dem 19. Jahrhundert wieder auf die ,athenische“ Form, das heißt einer auf Gemeinwohl und substanzielle Bürgertugenden bauende Staatstheorie, verkürzt worden, um sodann im Diskurs als, vormodern' marginalisiert zu werden (Skinner 1986, 1990, 1997). Philip Pettit setzt an Skinners Diagnose der halbierten Geschichte republikanischer Ideen an. Sein Erkenntnisinteresse ist jedoch kein begriffsgeschichtliches, sondern zielt auf die analytische Ausarbeitung des neo-römischen Republikanismus. Seiner umfassenden Darlegung in Republicanism - A Theory of Freedom and Government (Pettit 1999 a) - sowie deren freiheitstheoretischer Konkretisierung A Theory of Freedom: From the Psychology to the Politics of Agency (2001) - ließ er eine große Zahl von Aufsätzen folgen, welche die politik- bzw. rechtstheoretischen Konsequenzen erläutern und popularisieren halfen (anstatt vieler: Pettit 1999 b, 2000, 2004, 2008).

Dreh- und Angelpunkt des Neo-Republikanismus ist die Konzeption von Freiheit als Nicht-Beherrschung (non-domination). Dieser zufolge besteht Freiheit darin, vor willkürlichen Eingriffen durch andere Personen oder Institutionen geschützt zu sein. Der Neo-Republikanismus sei daher eine Alternative in zwei Richtungen: Gegen- 
über einem ,positiven' Freiheitsbegriff, welcher Freiheit kollektivistisch missverstehe, und gegenüber einem ,negativen' Freiheitsbegriff, der jede Form von Eingriff als Reduktion von Freiheit werte (Freiheit als non-interference). Der republikanische Freiheitsbegriff setze sich hiervon ab, da in ihm die Aufmerksamkeit auf staatstheoretische Überlegungen wie Rechtstaatlichkeit, Mischverfassung und Gewaltenteilung gelenkt wird. So werden praktische Fragen - wie die nach der Sicherung gerechter Sozialpolitik, demokratischer Legitimität oder dem Zuschnitt von Staatsaufgaben - unmittelbar freiheitsrelevant und sollen durch das institutionelle Arsenal republikanischer Theorie in innovativer Weise angegangen werden (Lovett/ Pettit 2009). Ein Angebot, welches durchaus ernst genommen wird, wie sich in den Bemühungen des ehemaligen spanischen Ministerpräsidenten José Zapatero zeigt, der Pettits Republicanism als Ausgangspunkt für seine politische Reformen zu nehmen versuchte und diesen mehrfach zu Vorträgen sowie zu einer Evaluation seiner ersten Amtszeit einlud (Martí/Pettit 2010 a).

In der Bugwelle der von Skinner und Pettit angeschobenen Arbeiten zum NeoRepublikanismus sind eine große Zahl von Publikationen zum Freiheitsbegriff entstanden. ${ }^{1}$ Auch die Frage, welche Institutionen das neo-republikanische Freiheitsideal am besten umzusetzen in der Lage sind, ist detailliert verhandelt worden (Bellamy 2007; Honohan/Jennings 2006). In Deutschland setzte die Rezeption verzögert ein, stieß aber auch auf viel Aufmerksamkeit (etwa Celikates 2010; Niederberger 2008; Hölzing 2013, 2014).

In der Aufregung um den Neo-Republikanismus sollte nicht übersehen werden, dass die Weiterentwicklung republikanischer Theorie auch aus anderen Richtungen Fahrt aufgenommen hat: Ein Beispiel ist die gegenwärtige französische Debatte um Denker wie Gauchet oder Rosanvallon (vgl. den Überblick bei Schulz 2011), ein zweites die an rechtstheoretische Lesarten Hannah Arendts anknüpfenden Untersuchungen (Thiel 2012; Volk 2013), ein drittes die Bezüge im Kontext agonistischer Demokratietheorien und Postdemokratie-Analysen (Niederberger 2009; Schaap 2009). Was eine republikanische Perspektive auf aktuelle Fragen von Politik und Gesellschaft ausmacht, was sie beinhaltet und was sie leisten kann, wird in diesen Ansätzen jeweils anders, aber doch unter gemeinsamen Bezugnahmen erörtert. Gerade weil Pettit selbst diese inner-republikanischen Weiterentwicklungen bislang nur sehr eingeschränkt aufgenommen hat, ist sein explizit politiktheoretisches Buch On the People's Terms: A Republican Theory and Model of Democracy (2012) mit besonderer Spannung erwartet worden. Im Folgenden unternehmen wir eine kriti-

1 Etwa Besson/Martí 2009; Laborde/Maynor 2008; Lovett 2010; Maynor 2003; Niederberger/Schink 2013; Viroli 2002. 
sche Rekonstruktion der dort vorgestellten Argumentation. Dabei soll insbesondere geprüft werden, inwiefern die Veränderungen, die Pettit dabei am bestehenden Theoriegebäude des Neo-Republikanismus vornimmt, den Diskussionen zu neueren Theorieentwicklungen des Republikanismus gerecht werden. ${ }^{2}$

On the People's Terms geht auf mehrere Vorlesungen (insbesondere die Seeley Lecture 2010, aber auch die Frankfurt Lecture 2011) zurück und stellt Pettits bisher stringentesten und weitreichendsten Versuch dar, die politische Theorie des NeoRepublikanismus zusammenzufassen. Er selbst stellt heraus, dass dieser Wurf nicht nur als Schärfung bestehender Argumente zu lesen ist, sondern zumindest phasenweise auch einschneidende Änderungen oder Revisionen beinhaltet. Weil Pettits Kernideen inzwischen einige Bekanntheit erlangt haben, kann er sich in On the People's Terms darauf konzentrieren, detailliert darzulegen, wie er die politischinstitutionellen Schwerpunkte republikanischer Theorie denkt. Das Buch wird daher systematisch in drei große Teile gegliedert. Diese bauen aufeinander auf, behandeln insgesamt aber eher getrennte Komplexe: Im ersten Teil geht es um die Konzeption von Freiheit als Nicht-Beherrschung (Einleitung und Kapitel 1, S. 1-74), im zweiten um soziale Gerechtigkeit und politische Legitimität als zentrale Ziele neo-republikanischer Politik (Kapitel 2 und 3, S. 75-186) und im dritten Teil wird der Entwurf eines neo-republikanischen Modells demokratischer Institutionen ausgearbeitet (Kapitel 4 und 5, S. 187-310).

\section{Republikanische Freiheit}

Einleitung und erstes Kapitel rekapitulieren das freiheitstheoretische Argument, demzufolge ein moderner Republikanismus zwar auf individuellen Freiheitsrechten aufbaut, auf Seiten der politischen Institutionen aber andere Wege geht als die liberale Theorie. Wie bereits in Republicanism ist es Pettit dabei ein Anliegen, jeden Verdacht zu zerstreuen, dass seine politische Konzeption einen ,Rückschritt' in kollektivistische, allein durch starke gemeinsame Moralvorstellungen zusammengehaltene Theoriekonzepte bedeuten könnte. Subjektive Individualität und gesellschaftliche Differenzierung werden als nicht hintergehbare Ausgangsbedingungen postuliert, was der Vision einer res publica aber nicht entgegenstehen müsse.

2 Philip Pettit hat im Frühjahr 2014 noch einen ergänzenden Band vorgestellt. Das in Just Freedom. A Moral Compass for a Complex World (Pettit 2014) vertretene Argument ist über weite Strecken identisch mit den Überlegungen in On the People's Terms. Wir machen zu den wenigen Erweiterungen von Just Freedom in den Abschnitten 2 und 3 kurze Anmerkungen, fokussieren ansonsten aber auf die die Wiedergabe des politikwissenschaftlich expliziteren On the People's Terms. 
Pettits erster Schritt ist es, eine Abgrenzung zu ,kommunitaristischen' oder ,populistischen' Versionen des Republikanismus zu vollziehen. ${ }^{3}$ Nicht nur verwirft er den starken und anspruchsvollen Tugendbegriff des ,klassischen' Republikanismus, der vom Einzelnen eine ständige und altruistisch motivierte Sorge um das Wohl aller verlange und diesen daher überfordere. Vielmehr konzentriert er seine Kritik auf das Problem ungeteilter Souveränität, zu welcher sich eine solche Konzeption stets verpflichte. Diese Kritik wird an Rousseau exemplifiziert und soll doch eine ganze Reihe von als Epigonen betrachteten Theoretikerinnen und Theoretiker von Kant bis Arendt treffen. Obwohl Rousseau rhetorisch ein Konzept von Freiheit als non-domination vertrete, bewirke seine Negation der institutionellen Dimension des Republikanismus (die kontestatorische Bürgerschaft und die Mischverfassung), dass der Freiheit der Boden entzogen werde. Rousseau verhelfe dem kollektiven Willen zu einer totalen Machposition, er entferne aus normativen Erwägungen die prozeduralen Schutzvorkehrungen, derer die Republik bedürfe. Innerhalb des argumentativen Aufbaus von On the People's Terms ist diese Diskussion insofern wichtig, als dass Pettit damit seine eigenen Ausführungen über die Kontrolle und Teilung des Regierungsapparats und seinen spezifischen Vorschlag zu republikanischer Bürgertugend vorbereitet. Wie schon in früheren Texten handelt es sich aber letztlich um nicht viel mehr als die polemische Zurückweisung eines überzeichneten Gegners (Bellamy i. E.). ${ }^{4}$

Ganz anders stellt sich das Verhältnis zu den liberalen Freiheitskonzeptionen dar: Von dieser Seite wurde Pettit zuletzt wieder und wieder mit dem Einwand konfrontiert, dass sich seine Theorie nicht - wie behauptet - kategorisch vom Liberalismus unterscheiden lasse (Carter 2007; Kramer 2007). Und tatsächlich betont Pettit selbst ja, dass die republikanische Freiheit eine Variante der ,negativen' Freiheit sei, allerdings eine dem liberalen Verständnis deutlich überlegene, da für Dominanzsituationen sensible. Im Zentrum der Auseinandersetzung mit diesen Theorien in On the People's Terms steht deshalb die Frage, wie sich die Unterscheidung zwischen interference und domination exakter treffen lasse. Dass bereits die Po-

3 Schon früher hatte Pettit den Neo-Republikanismus als, dritten Weg ' jenseits von Liberalismus und Kommunitarismus zu verorten gesucht (vgl. etwa Pettit 1994). Die Abgrenzung zum Kommunitarismus findet sich sogar noch ausführlicher in einem Beitrag in Niederberger/Schink 2013 (Pettit 2013), was als Indiz dafür gedeutet werden kann, dass Pettit die Abgrenzung in dieser Richtung zunehmend wichtiger wird als die kategoriale Unterscheidung zu den liberalen Konzeptionen.

4 Mit dieser unterkomplexen Kritik republikanischen Denkens steht Pettit keinesfalls allein da; auch von anderen Vertretern des Neo-Republikanismus wie Quentin Skinner oder Frank Lovett wird zwar viel Zeit und Raum darauf verwendet, die theoretische Eigenständigkeit und Spezifizität zu betonen, eine ernstzunehmende Verortung neo-republikanischer Konzepte innerhalb der breiteren Republikanismus-Tradition fehlt - wohl aus theoriestrategischen Gründen - aber völlig. 
tentialität eines Eingriffes Probleme mit sich bringt (, domination without interference), ist einleuchtend (und lässt sich beispielsweise an der im Anschluss an die Snowden-Leaks geführten Kontroverse um geheimdienstliche Datensammlung gut erörtern). Es fällt jedoch auf, dass es für die Abgrenzung von liberalen Theorien mindestens ebenso entscheidend sein sollte, die umgekehrte Relation herauszuarbeiten, nämlich, dass nicht jede Art von interference eine Einschränkung der Freiheit bedeuten muss (,interference without domination ). Diese Erörterung fällt jedoch auch in den beiden aktuellen Büchern extrem knapp aus: So betont Pettit, dass interference genau dann als nicht-beherrschend gelten darf, wenn sie geeigneter Kontrolle unterliegt. Weil nun aber die institutionellen Schlussfolgerungen, die aus der Konzeption von Freiheit als non-interference respektive non-domination gezogen werden, sich teilweise bis zur Ununterscheidbarkeit gleichen (dazu unten mehr), wäre ein stärkeres Argument nötig, um wirklich einen kategorialen Unterschied behaupten zu können. Hier aber gibt es nur den dünnen Versuch, festzuhalten, dass die republikanische Seite der Unterscheidung intuitiver zu begründen sei - und daher bessere Chancen habe, sich in modernen, pluralistisch verfassten Gesellschaften durchzusetzen (vgl. auch Martí/Pettit 2010 b) - ein Argument, dass deklarativ und zirkulär anmutet und der Stellung als Schlüssel für die folgenden institutionentheoretischen Überlegungen nicht gerecht wird.

Überhaupt bewirkt die Fokussierung auf den Unterschied von domination und interference eine Engführung, die Pettit den Blick für viele Probleme verstellt, die in modernen Freiheitstheorien prominent diskutiert werden, so zum Beispiel die Subjektivierungsproblematik. Das Verpassen strukturierender Wirkungen gesellschaftlicher Ordnung und die Konzentration auf inter-personale Beziehungen erklärt beispielsweise auch die zögerlich-abwartende Reaktion, die feministische Theoretiker mit Blick auf den Erfolg neo-republikanischer Theorie an den Tag legen (Phillips 2000; Friedman 2008).

Drittens muss im Kontext der Debatte um Freiheit als Nicht-Beherrschung auch noch der normativen Status der Unterscheidung diskutiert werden. Pettit zufolge handelt es sich bei seiner Konzeption um eine nicht-normative Sichtweise (etwa Pettit 2006 b). Dies ist wichtig, da er auf dieser Grundlage annimmt, dass sich (potenzielle) Fälle von Beherrschung objektiv identifizieren lassen und sich entsprechend unstrittige institutionelle Maßnahmen gegen sie finden werden (Pettit 2012: $57 \mathrm{ff}$.). Doch lässt sich zum einen fragen, ob es nicht-normative Konzeptionen von Beherrschung überhaupt geben kann (vgl. Busen i. E.), zum anderen einwenden, dass weil für Pettit eben nicht jede Form von interference Beherrschung darstellt, diese Unterscheidung (bzw. die Bestimmung der relevanten Kontrollmechanismen) nur im Rückgriff auf bestimmte (und zwar liberale) Prinzipien wie beispielsweise 
die gleiche Achtung von Individuen vorgenommen werden kann (Carter 2000; Christman 1998; Costa 2007; Larmore 2003; McMahon 2005). Pettits vorgeblich neutrales Argumentieren für nicht-beherrschende, aber liberal anmutende Institutionen ist dabei selbst innerhalb des Neo-Republikanismus infrage gestellt worden. Frank Lovett etwa gelangt aus einer sich ebenfalls als nicht-normativ verstehenden Konzeption zu ganz anderen, stärker mit liberalen Theorien kontrastierenden Empfehlungen - unter anderem der Einführung eines bedingungslosen Grundeinkommens (vgl. Lovett 2010, Kapitel 7). Statt sich mit diesen Einwänden detailliert auseinanderzusetzen und das Potenzial des neo-republikanischen Theorieprogramms, das die originellen Vorschläge von Lovett und anderen andeuten, weiter auszuleuchten, belässt es Pettit bei einer knappen Zurückweisung der entsprechenden Kritik und weist darauf hin, dass etwa zwischen seiner und anderen neueren neorepublikanischen Theorien nur kleine, letztlich empirische Unterschiede bestünden (Pettit 2012: 58 f.).

\section{Republikanische Gerechtigkeit}

Der zweite Komplex von On the People's Terms dürfte für das meiste Aufsehen sorgen: In ihm wendet sich Pettit dem sich nie so recht erschöpfenden Gerechtigkeitsdiskurs zu und stellt einen spezifisch republikanischen Blick auf diesen in Aussicht. Grundlegend hierfür ist seine kategoriale Unterscheidung von justice (als ein zwischen den Bürgern entstehendes Gut) und legitimacy (welche ein Gut zwischen dem Staat und seinen Bürger sei). Mit dieser Unterscheidung vertieft Pettit die schon in früheren Texten getroffene Unterscheidung von dominium und imperium als den zwei Kontexten, in denen Beherrschung entstehen kann. Nur in wenigen Strichen ausgewiesen, aber von potenziell enormer Sprengkraft ist dabei seine Behauptung, dass es einer republikanischen Theorie vorrangig um legitimacy und weniger um justice gehen müsse. Der Grund hierfür wird mehr angedeutet als ausgeführt: Es ist Pettits Annahme, dass die Fähigkeit des Staates zur Unterdrückung - das heißt dessen überlegene, souveräne Macht - diesen zur eigentlichen Bedrohung von Freiheit mache. Für eine solche Diagnose sprechen zwar Beobachtungen wie die Ausweitung staatlicher Regulierungsmacht auf immer neue Politikfelder, doch inwiefern es überzeugend ist, in staatlicher Macht auch heute noch ein solches Potenzial zu tiefgreifenden und gesellschaftlich nicht zu konternden Eingriffen zu sehen und ihr gerade im Vergleich zu korporativen privaten Akteuren eine so absolute Sonderstellung zuzuschreiben, hätte zumindest mit einem Fragezeichen versehen und einer eingehenden empirischen Analyse zugeführt werden müssen. Hier gehen andere republikanische Arbeiten deutlich weiter und versuchen das Verhält- 
nis zwischen Beherrschung und sozialer Macht gezielt auszuloten (Lovett 2010; Pansardi 2013). Moderne Gesellschaften sind auch oder sogar gerade durch Beherrschungsverhältnisse jenseits von staatlicher Regulierung und individuellen Grundrechten geprägt und die Fixierung auf die Kontrolle des Staates (und nochmals zugespitzt auf die potenzielle Gefahr der Mehrheitsherrschaft) bekundet ein gesellschaftstheoretisches Desinteresse, welches der vielleicht augenfälligste Anachronismus von Pettits Theorie ist. Ein Anachronismus, der zudem deshalb besonders ins Gewicht fällt, weil er ein Unverständnis für komplexe Gesellschaften bekundet, welche Pettit doch eigentlich mit der klassischen republikanischen Sprache versöhnen will.

Aus systematischer Sicht wirft die Trennung von Gerechtigkeit und Legitimität die Frage auf, wie deren Verhältnis zu konzipieren ist. Dieses steht im Mittelpunkt einer bereits länger anhaltenden Debatte einer Vielzahl republikanischer Autoren um das Verhältnis von demokratischer Politik, Recht und Verfassung - mit einem besonderen Fokus auf der Institution des judicial review (vgl. die Beiträge in Besson/ Martí 2009). Zentraler Streitpunkt ist, inwiefern eine das republikanische Freiheitsideal verbürgende Ordnung bestimmte Rechte dergestalt sichern muss, dass sie dem demokratischen Zugriff ,normaler' Politik entzogen bleiben. Die eine Seite (darunter etwa Bellamy 2007, 2009; Waldron 2004, 2006) geht von der Priorität der Selbstherrschaft aus, weshalb Recht und dessen Interpretation letztinstanzlich der Demokratie klar untergeordnet werden müsse. Bedenken wie die einer Tyrannei der Mehrheit ließen sich durch prozedurale Justierungen hinreichend ausräumen. Die andere Seite teilt diesen Optimismus bzgl. demokratischer Verfahren nicht und insistiert darauf, dass individuelle Freiheit nur durch Schutzrechte gesichert werden kann (Sager 2014). Demokratie und Verfassungsgerichtsbarkeit werden hier als notwendige Einheit gesehen (etwa Honohan 2009, aber auch schon Michelman 1988; Sunstein 1999). Die Debatte macht deutlich, dass in der republikanischen Theorie das Spannungsverhältnis zwischen Recht bzw. Gerechtigkeit und Demokratie (und damit: zwischen individueller und kollektiver Autonomie) eine hohe Virulenz besitzt und eine Vielzahl institutioneller Vorschläge existiert. Umso mehr erstaunt, dass Pettit in On the People's Terms ohne weitere Begründung explizit erklärt, zu diesem Verhältnis keine Position beziehen zu wollen (Pettit 2012: 24 f.). In der Darstellung der beiden neo-republikanischen (Teil-)Projekte der Realisierung von justice und legitimacy kommt er daher ohne jeden Verweis auf deren Verbindungslinien und Spannungsmomente aus. Somit aber legt Pettit streng genommen zwei unabhängige Theorien neo-republikanischer Politik vor, nämlich eine Gerechtigkeits- und eine Demokratietheorie. Zwar liegt beiden das neo-republikanische Ziel der Verhinderung von Beherrschung zugrunde, dieses wird aber sowohl be- 
gründungstheoretisch unterschiedlich ausbuchstabiert als auch in sehr unterschiedliche und zumindest potenziell konträre institutionelle Schlussfolgerungen überführt.

Die Vermeidung von private domination, das heißt von Zuständen der Beherrschung durch Individuen bzw. Gruppen, verlangt nach Pettit, dass für den Einzelnen ein klar abgegrenzter Bereich persönlicher Autonomie gesichert werden muss. Entsprechend fragt er danach, welches Set von individuellen Entscheidungsmöglichkeiten in Form von basic liberties geschützt werden sollte. So soll jedem gleichermaßen die Verwirklichung eines individuell als bedeutungsvoll betrachteten Lebens möglich werden. Nicht nur auf den ersten Blick erinnert dies stark an liberale Gerechtigkeitstheorie (vgl. Rawls' Frage nach dem ,umfangreichste[n] System gleicher Grundfreiheiten [...], das mit dem gleichen System für alle anderen verträglich ist" (Rawls 1979: 81)). Für Pettit besteht der entscheidende Unterschied nun aber darin, dass seine Konzeption von basic liberties einen konstitutiven Rückgriff auf das Ideal des „liber“ macht, des freien Bürgers, der seinen gleichermaßen freien Mitbürgern im öffentlichen Leben auf Augenhöhe begegnen kann. Die Anwendung des daraus abgeleiteten, ,eyeball-test“ kulminiert in der Frage, welcher Schutzrechte und Ressourcen es für eine Person bedarf, um mit ihren Mitbürgerinnen ohne Angst oder Unterwürfigkeit interagieren zu können (Pettit 2014: 98-101). Die so formulierte Heuristik ist überzeugend und prägnant und erlaubt eine kontextsensible und an der gesellschaftlich-sozialen Realität orientierte Abwägung von Sicherungsmechanismen. Irritierend ist jedoch, dass Petitt diesen ,eyeball-test“" offenbar als rein theoretisches Instrument versteht - eine Art Gedankenexperiment, das sich unabhängig von tatsächlichen ,Begegnungen' anwenden lässt. Entsprechend abstrakt und allgemein sind die basic liberties, die er in seinen beiden jüngsten Büchern als zu vermutendes Ergebnis des Test formuliert (Pettit 2012: 103, 2014: 72). Auch diesbezüglich gilt, dass im republikanischen Feld selbst eine Vielzahl sehr viel konkreterer und originellerer Vorschläge zu finden wären - etwa hinsichtlich wohlfahrtsstaatlicher Arrangements (Cassassas/De Wispelaere 2012; Halldenius 1998; Lovett 2009, Kap. 7) oder dem Umgang mit ethnischen und religiösen Minderheiten (Laborde 2008), Flüchtlingen und Migranten (Honohan/Hovdal-Moan 2014) sowie Menschen mit Behinderungen (Cassassas/De Wispelaere 2013). Pettit erscheint hier unentschieden zwischen einem eher theorie- und einem eher praxisbezogenen $\mathrm{Zu}$ gang - worin sich auch plastisch das ungelöste Spannungsverhältnis zwischen Gerechtigkeit und Demokratie spiegelt. Primär theoretisch gewonnene Vorschläge zur Realisierung des neo-republikanischen Freiheitsideals, wie sie die genannten Ausführungen zum ,eyeball-test" darstellen, laufen dabei erkennbar Gefahr, dass sie als ,unpolitische' Konstruktionen abgetan werden (eine erstaunliche, wenn auch nicht 
zufällige Parallele zu Habermas' Kritik an Rawls' Prinzipien der Gerechtigkeit (vgl. zur Übersicht Finlayson/Freyenhagen 2011)). Sollen dagegen, wie Pettits Überlegungen an anderer Stelle suggerieren, die konkreten Realisierungsformen zum Schutz individueller Freiheit jeweils im Lichte der sozio-kulturellen Spezifika unterschiedlicher Gesellschaften praktisch festgelegt (und reformiert) werden (Pettit 2012: 263, 2014: xxvii), muss spezifiziert bzw. kritisch beleuchtet werden, auf welche Art und Weise dies genau geschehen kann oder soll. Wenn sich, wie Pettit andeutet, die rechtsförmig zu schützenden Möglichkeitsräume hier letztlich ebenfalls mithilfe des hypothetischen ,eyeball-test“ - sei es durch den politischen Theoretiker oder andere ,Experten " - abstrakt bestimmen lassen (oder als gewissermaßen intuitiver Konsens aller Bürgerinnen vorausgesetzt werden), bedarf es auch hier keines Gesprächs über die res publica. Politische Kämpfe etwa um die Definition oder Interpretation der in einer Gesellschaft zu verwirklichenden Grundrechte und -freiheiten haben dann keinen eigenen Ort und republikanische Gerechtigkeit droht zu einem Eliten-Projekt zu werden (McCormick 2006, 2007, 2013).

\section{Republikanische Demokratie}

Damit sind wir im dritten und letzten Teil des Buches angelangt, welcher sich als eigenständige neo-republikanische Demokratietheorie lesen lässt (worin auch für Pettit der besondere Mehrwert des Buches liegt). Im Mittelpunkt steht hier die Identifizierung jener Mittel, die geeignet sind, zu verhindern, dass die notwendigen korrigierenden Eingriffe - mithin: die Herrschaft - des Staates einen beherrschenden Charakter annehmen.

Im Gegensatz zu den relativ statisch bestimmten basic liberties und in einer zumindest teilweisen Abkehr von früheren Schwerpunktsetzungen im Legitimitätskonzept (Pettit 2000, 2004), ist Pettit hier sehr darum bemüht, die , politische' Natur der Kontrolle (und entsprechend die Rolle eines aktiven bürgerschaftlichen Handelns) herauszustellen. Damit reagiert er offenbar auf die häufig geäußerte Kritik, dass seine Konzeption zu eindimensional rechtsstaatlich angelegt sei und die Demokratie nur rhetorisch im Namen führe. Theoriestrategisch äußert sich dies darin, dass zur Beurteilung der demokratischen Qualität eines Gemeinwesens nun explizit zwei gleichwertige Kriterien herangezogen werden: erstens, dass staatliches Handeln gleichermaßen durch die Interessen aller Bürgerinnen informiert wird (, democratic influence ), und zweitens, dass die Bürger sich gleichzeitig (und gleichermaßen) selbst kontrollieren, so dass allgemeinverbindliche Entscheidungen sich ausschließlich am so gewonnen Allgemeininteresse orientieren (, democratic control ${ }^{~}$ ). Hierin ist zwar angelegt, dass Pettit auf den institutionellen Rahmen konzentriert 
bleibt und es sich sich bei der demokratisch vermittelten Herstellung eines Allgemeininteresses (public interest) letztlich um eine Vermittlung/Versöhnung individueller Privatinteressen handelt (liberale Topoi also nach wie vor sehr stark sind), doch da wir diese Kritik bereits mit Blick auf die ersten beiden Teilen entwickelt haben, wollen wir mit Blick auf diesen dritten Teil allein die Änderungen im Verständnis republikanischer Bürgerschaft betrachten.

Zwei Aspekte stechen bei Pettits ,Umbauarbeiten“ besonders hervor: die Aufwertung eines einheitlichen Verständnisses von Bürgerschaft und die Anmerkungen zum politischen Engagement in der kontestatorischen Bürgergesellschaft. Beide reagieren auf den Vorwurf einer unterentwickelten Demokratietheorie und versuchen den Spagat zu bewerkstelligen, sowohl die Bedeutung kollektiven Handelns herauszustreichen ohne die Segnungen negativer Freiheitsrechte zu gefährden. Pettit wendet sich daher nach wie vor konsequent gegen von Mehrheiten dominierte Kollektiventscheidungen, glaubt aber zeigen zu können, dass aus seinem Verständnis von Demokratie dennoch ein handlungsfähiges Kollektivsubjekt erwachsen kann. Dieses wird an einer schon irritierend großen Zahl von Stellen beschworen und immer wieder mit Formulierungen eingeführt, die der Gesamtheit der Bürgerschaft einheitliche Präferenzen und Intentionen zuschreiben. Der Text schließt gar mit einer Auseinandersetzung zum Vorrang der constituting people vor den constituted people (etwa assoziierte Bürger vs. instiutionalisierte Regierung), was eher an Ingeborg Maus oder Bruce Ackermann (und damit an Rousseau und Kant erinnert) erinnert als an die liberale Tradition starker Rechte und nur zu deren Schutz errichteter Institutionen. Insofern überrascht es nicht, dass zuletzt aus Kantianischer Perspektive verschiedentlich versucht worden ist, Pettit und seine Theorie ,einzugemeinden' (etwa Forst 2013). Die lange sehr prominente Unterscheidung von auktorialer und editorialer Dimension wird dagegen in On the People's Terms nur noch an einer einzigen Stelle kurz wiederholt; die Vorstellung einer pluralen Bürgerschaft spielt dementsprechend fast keine Rolle mehr.

Um überzeugend die Herausbildung eines solchen starken Kollektivsubjekts in Aussicht stellen zu können ohne dabei auf den klassischen Republikanismus mit seiner Tugend- und Gemeinwohlorientierung zurückzugreifen, muss Pettit Annahmen darüber aufstellen, wie sich aus einer individuell motivierten politischen Praxis unter demokratischen Bedingungen als ,by-product ' allgemein anerkannte und geteilte Normen hervorbringen lassen. Letztere sollen nämlich selbst oder sogar gerade dann entstehen, wenn es zu einem individuell motivierten Anfechten (contestation) von Regierungsentscheidungen kommt. Ein solches könne nämlich nur dann überhaupt Aussicht auf Erfolg haben, wenn allgemein teilbare Gründe/Normen für den Akt der Kontestation bzw. deren Zurückweisung vorgebracht werden. Hierdurch 
kristallisiere sich das gemeinsame Fundament eines Gemeinwesens immer weiter heraus. Mit einer solchen Argumentation kommt Pettit deutlich in die Gefilde deliberativer Demokratietheorie - so nah, dass er sich schließlich selbst zu einer Erklärung genötigt sieht, inwiefern sich seine republikanische Demokratietheorie von dieser noch unterscheidet. Die von ihm vorgeschlagene Abgrenzung - dass nämlich seine Theorie nicht nur solche Entscheidungen als legitim ansehe, die Ergebnis tatsächlicher Deliberation sind - ist aber weder mit Blick auf den aktuellen Stand der deliberativen Demokratietheorie noch bezüglich der Passung zu seiner eigenen Argumentationsentwicklung überzeugend. Aufschlussreich ist sie aber trotzdem, und zwar insofern als sich hier deutlich zeigt, dass auch ein demokratisch gewendeter Pettit noch zur Entpolitisierung des Demokratiebegriffs neigt: „The impact of deliberative norms may be elusive, indeed almost invisible, if the system is working well. Assuming that it is working at its best, the institutions established will not be called into question [...]" (Pettit 2012: 268). Aktive Einflussnahme auf politisches Entscheidungshandeln bzw. dessen kritisches Infragestellen sind in dieser Konzeption von Demokratie, wenn alles gut läuft, also gar nicht nötig; die reine Etablierung institutioneller Vorkehrungen, die eine solche Praxis grundsätzlich ermöglichen würden, reicht aus, um die Regierenden auf der richtigen Bahn zu halten. ${ }^{5}$

Diese grundsätzliche Entscheidung muss auch im Blick behalten werden, wenn wir uns der anderen großen Baustelle neo-republikanischer Demokratietheorie zuwenden: der kontestatorischen Bürgerschaft. Diese ist als zentrales Element republikanischer Demokratie bereits in den frühesten Ausarbeitungen von Pettits Republikanismus ausgeflaggt (vgl. insbesondere Pettit 2000), doch wurde in Republicanism und anderen Schriften nur wenig zu der aktiven Rolle der Bürger gesagt, sondern der Fokus allein auf die institutionelle Rahmung gelegt. On the People's Terms geht hierüber hinaus und versucht klarer zu benennen, wie aktive Eingriffe aussehen und welcher Wert ihnen zukommt. Auch überwindet Pettit damit die Eng-

5 Es passt in dieses Bild, dass Pettits Ausführungen zu den institutionellen Realitäten sehr abstrakt und unterdeterminiert bleiben. Kaum einmal versucht er, den diagnostischen Nutzen seiner Ausführungen nachzuweisen oder seine Theorie durch empirische Beobachtung zu schärfen, wie dies für so viele Klassiker republikanischer Theorie typisch ist. Vielmehr verweist er auf eine Arbeitsteilung zwischen ,Theorie' und ,Praxis' und nimmt damit seine Ankündigung, eigene Vorschläge zu machen und Relevanz nachzuweisen, faktisch wieder zurück.

Dieser Umstand spiegelt sich auch in der Sprache seiner Ausarbeitung, wo Pettit in einer aufdringlichen und sich durch alle Teile des Textes durchziehenden Weise mit systematisch-analytischen Gedankenexperimenten argumentiert (von betrunkenem Autofahren bis zur Wahl von Restaurantoptionen), welche dann in Analogie zur politischen Sphäre gesetzt werden. Einer solchen argumentativen Strategie fehlt nicht nur die Verve, die weite Teile der republikanischen Tradition kennzeichnen, sie verhindert auch, dass die Theorie auf dem Höhepunkt ihrer Rezeptionswelle zu einem bedeutenden Spiegel gegenwärtiger Politik werden könnte. 
führung auf das Moment der Wahl, welches vorher weitgehend synonym mit seinem Verständnis demokratischer Einflussnahme schien. Als Beispiel kann seine Behandlung von Opposition und zivilem Ungehorsam dienen, die er unter anderem unter Hinweis auf Bonnie Honig und Iris Marion Young zu bestimmen versucht (2012: 225 ff.). Mit der Bejahung ungeregelter zivilgesellschaftlicher Eingriffe und des dynamischen Moments einer aktiven Bürgerschaft geht jedoch noch im selben Abschnitt einher, dass er die Vorrangigkeit der funktionalistischen Beschreibung der Rolle politischer Auseinandersetzung (watchdog) unterstreicht. Was Widerspruch jenseits der Verletzung von individuellen Interessen motivieren könnte, scheint für ihn nicht weiter interessant. Vielmehr ist es so, dass sein expliziter Entschluss, auf einen starken Tugendbegriff zu verzichten und an dessen Stelle das Moment der civic vigilance zu setzen, systematisch verhindert, dass Motivationen für politisches Handeln sichtbar würden, die über ein strategisches Eigeninteresse hinausgehen. Gerade soziale Bewegungen sind jedoch nur zu verstehen, wenn sie nicht als allein strategisch-interessenrationale Gruppierungen analysiert werden, sondern die gewählten Ausdrucksformen ernst genommen werden. Wie Pettits Vorstellung von der Funktion der civic vigilance - die sich arbeitsteilig und Emotionen filternd vollziehen soll, so dass der Handlungsdruck vom Individuum genommen und die Zivilisiertheit politischer Äußerungen sichergestellt ist - deutlich macht, stellen in seiner Konzeption ernsthafte politische Auseinandersetzungen letztlich vor allem einen Hinweis auf das Versagen der Ordnung dar (eine überzeugendere, da stärker historisch argumentierende Rekonstruktion kontestatorischer Bürgerschaft präsentiert Pierre Rosanvallon $(2009,2010)$; auf die generellen Schwierigkeiten dergestalt , unpolitischer' Konzeptionen hat Nadia Urbinati (2010) hingewiesen).

Auch die Passagen zum Widerstandsrecht unterstreichen dies: So spricht Pettit sich gegen allzu revolutionäre Umtriebe aus, da ihm zufolge Staaten quasi als Systemeigenschaft Legitimierbarkeit zukommt (2012: 139 ff.). Dieses Potenzial sei zu achten, weswegen Reform und nicht Revolution das Mittel der Wahl sei. Dass er wenig später einen positiv konnotierten Resistenzlevel einer jeden Gesellschaft einführt (ebd.: 174), nimmt dies nicht zurück, sondern wird wieder funktionalistisch als eine Art Barometer über die Unzufriedenheit der Regierten mit der Regierungsführung gedacht. Somit bleibt Widerstand analog zu der liberalen Logik einzig eine ,letzte Option', um die Herrschenden zu einem erneuten In-Kraft-Setzen der Selbstbindung aufzufordern. Die gesellschaftlichen Kontexte verschwinden völlig, und dass die politische Äußerung von Kritik ihren Ursprung nicht nur im Staat-Bürger- 
Verhältnis hat, sondern (wenn nicht gar häufiger) aus sozialen Fragen entspringt, lässt sich in seiner Theorieanlage kaum nachvollziehen. ${ }^{6}$

Auch von der aktualisierten, ausführlicheren und auf den ersten Blick im Vergleich zu früheren Texten sehr viel ,politischeren' Fassung einer republikanischen Demokratietheorie lässt sich also behaupten, dass sich mittels ihrer kaum nachvollziehen lässt, wie politische Auseinandersetzung sich strukturiert, welche Dynamik politische Prozesse antreibt und wie Meinungsbildung jenseits institutioneller Prozeduren verläuft. Genau dies ist in den vergangenen Jahren allerdings, insbesondere durch den Einfluss radikaldemokratischer Theorien, zu einem der wichtigsten Forschungsgebiete in der Demokratietheorie geworden. Dass radikaldemokratische Ansätze dabei durchaus ins Gespräch mit modernen republikanischen Ansätzen gesetzt werden können und es eine gewisse Schnittmenge bei den rezipierten Autoren gibt, hat etwa jüngst Christian Volk (2013) zu zeigen versucht. Pettits weitestgehend auf die Befürwortung von Gewaltenteilung und reflexiver Deliberation gegründetes Demokratiemodell ist gegenüber einer solchen, die Bedingungen pluralistischer Politik und politischen Handelns reflektierenden Position diagnostisch unterlegen. Weil er sich nicht die Mühe macht, das von ihm vorgeschlagene Amalgam von institutionellen Arrangements auch auf potenzielle Dysfunktionalitäten, Widersprüchlichkeiten und negative Pfadabhängigkeiten zu befragen, wird sein Modell republikanisch-demokratischer Ordnung bei allem vorgeblichen Realismus utopistisch und ist allenfalls zufällig stabil.

Eine letzte kleine Enttäuschung ist schließlich, dass Pettit in On the People's Terms darauf verzichtet, die wachsende Bedeutung internationaler Politik in $\mathrm{Zu}-$ sammenhang zu seiner Demokratietheorie zu stellen - obwohl er zuvor zumindest Andeutungen bzgl. des Potenzials des Neo-Republikanismus jenseits staatlicher Politik gemacht hatte (Petitt 2006, 2010) und in diesem Kontext durchaus stark rezipiert wird (z. B. Keohane/Macedo/Moravcsik 2009). Just Freedom stellt in dieser Hinsicht eine Erweiterung dar, enthält es doch explizit ein Kapitel zur internationalen Dimension. Hier versucht sich Pettit jedoch vornehmlich an einer Bestimmung eines bedingten, aber sehr weitreichenden Souveränitätsprinzips, was der großen Spannweite republikanischer Deutungsansätze internationaler Politik erneut nur sehr unzureichend Rechnung trägt. ${ }^{7}$

6 Eine Kritik, die vor allem auch durch John P. McCormick $(2011,2013)$ formuliert wird. Dieser argumentiert ideengeschichtlich gegen Pettit und Skinner, dass die machiavellische Demokratie sich weit eher um das Austragen sozialer Kämpfe als um die institutionelle Sicherung der Rationalität rechtlich formierter Institutionen gekümmert habe.

7 Vergleiche etwa Marchart 2005; Deudney 2008; Laborde 2010; Thiel 2012; Gädeke 2014. 
Unter dem Strich haben sich Pettits in den beiden jüngsten Büchern vorgenommene Renovierungsarbeiten also eher als kosmetische Eingriffe erwiesen. Zentrale und in der Diskussion mittlerweile nachdrücklich aufgedeckte Schwachstellen des Neo-Republikanismus werden durch die Reformulierung nicht behoben, sondern bestenfalls kaschiert. Pettit weicht der Auseinandersetzung mit Kritikern tendenziell aus und versucht Anschlussfähigkeit durch eine Umarmungsstrategie herzustellen. Hierdurch schleifen sich die Kanten jedoch immer weiter ab und wird die Nähe zum Mainstream der angloamerikanischen liberalen Theorie absolut augenfällig.

Mit diesem harten Urteil über die mangelhafte Fortentwicklung republikanischer Demokratietheorie durch Philip Pettit ist jedoch noch nicht der Stab über die Entwicklung, Aktualität und Relevanz republikanischer Theorien als Ganzer gebrochen. Der Blick über Pettit hinaus zeigt, dass republikanische Ansätze in der gegenwärtigen demokratietheoretischen Debatte relevant und anschlussfähig sind. Politiktheoretische und gesellschaftstheoretische Ansätze, die nicht so sehr die analytische Freiheitskonstruktion in den Blick rücken, sondern an einer zeitgemäßen Reformulierung der Bedingungen demokratischer Politik interessiert sind, greifen das zentrale republikanische Anliegen, die Pluralität von Gesellschaft durch politisches Handeln zu erschließen, in einer Weise auf, die eine echte Alternative zum liberalen Mainstream darstellen kann.

\section{Literatur}

Bellamy, Richard, 2007: Political Constitutionalism: A Republican Defence of the Constitutionality of Democracy, Cambridge.

Bellamy, Richard, 2009: The Republic of Reasons. Public Reasoning, Depoliticization, and Non-Domination, in: Samantha Besson/José Luis Martí (Hrsg.), Legal Republicanism. National and International Perspectives, Oxford, 102-120.

Bellamy, Richard, i. E.: A Modest Defence of neo-Rousseauvian Republicanism, in: Political Theory.

Besson, Samantha/Martí, José Luis (Hrsg.), 2009: Legal Republicanism. National and International Perspectives, Oxford.

Busen, Andreas, i. E.: Non-Domination, Non-Normativity, and Neo-Republican Politics, in: Critical Review of International Social and Political Philosophy.

Carter, Ian, 2000: A Critique of Freedom as Non-Domination, in: The Good Society 9, 42-46.

Carter, Ian, 2008: How are Power and Unfreedom related?, in: Cécile Laborde/John Maynor (Hrsg.), Republicanism and Political Theory, Oxford, 58-82. 
Cassassas, David/De Wispelaere, Jurgen, 2012: The Alaska Model. A Republican Perspective, in: K. Widerquist/M. W. Howard (Hrsg.), Alaskas Permanent Fund Dividend. Examining its Suitability as a Model, New York, 169-188.

Cassassas, David/De Wispelaere, Jurgen, 2013: A Life of One's Own. Republican Freedom and Disability, in: Disability \& Society 29 (3), 402-416, DOI: 10.1080/09687599.2013.823076.

Celikates, Robin, 2010: Republikanismus zwischen Politik und Recht. Ein Literaturbericht, in: Zeitschrift für philosophische Forschung 64 (1), 111-129.

Christman, John, 1998: Review of Republicanism, in: Ethics 109, 202-206.

Costa, M. Victoria, 2007: Freedom as Non-Domination, Normativity, and Indeterminacy, in: The Journal of Value Inquiry 41, 291-307.

Deudney, Daniel, 2008: Bounding Power. Republican Security Theory from the Polis to the Global Village, Princeton.

Finlayson, James Gordon/Freyenhagen, Fabian, 2011: Habermas and Rawls. Disputing the Political, London.

Forst, Rainer, 2013: A Kantian Republican Conception of Justice as Non-Domination, in: Andreas Niederberger/Philipp Schink (Hrsg.), Republican Democracy. Liberty, Law and Politics, Edinburgh, 154-168.

Friedman, Marilyn, 2008: Pettit's Civic Republicanism and Male Domination, in: Cécile Laborde/John Maynor (Hrsg.), Republicanism and Political Theory, Oxford, 246-268.

Gädeke, Dorothea, 2014: Politik der Beherrschung. Zur ambivalenten Universalisierung der Demokratie, unveröffentl. Dissertation, Frankfurt a. M.

Halldenius, Lena, 1998: Non-Domination and Egalitarian Welfare Politics, in: Ethical Theory and Moral Practice 1, 335-353.

Honohan, Iseult/Jennings, Jeremy, 2006: Republicanism in Theory and Practice, London/New York.

Honohan, Iseult, 2009: Republicans, Rights, and Constitutions. Is Judicial Review Compatible with Republican Self-Government?, in: Samantha Besson/José Luis Martí (Hrsg.), Legal Republicanism. National and International Perspectives, Oxford, 83-101.

Honohan, Iseult/Hovdal-Moan, Marit (Hrsg.), 2014: Special Issue: Domination, Migration and Non-Citizens, in: Critical Review of Social and Political Philosophy 17 (1).

Hölzing, Philipp, 2013: Republikanismus und Kosmopolitismus, Frankfurt a. M./ New York.

Hölzing, Philipp, 2014: Republikanismus. Geschichte und Theorie, Stuttgart. 
Keohane, Robert O./Macedo, Stephen/Moravcsik, Andrew, 2009: Democracy-Enhancing Multilateralism, in: International Organization 63 (1), 1-31.

Kramer, Matthew, 2008: Liberty and Domination, in: Cécile Laborde/John Maynor (Hrsg.), Republicanism and Political Theory, Oxford, 31-57.

Laborde, Cécile, 2008: Critical Republicanism. The Hijab Controversy and Political Philosophy, Oxford.

Laborde, Cécile, 2010: Republicanism and Global Justice: A Sketch, in: European Journal of Political Theory 9 (1), 48-69.

Laborde, Cécile/Maynor, John, 2008: Republicanism and Political Theory, Oxford. Larmore, Charles, 2003: Liberal and Republican Conceptions of Freedom, in: Critical Review of International Social and Political Philosophy 6 (1), 96-119.

Lovett, Frank, 2010: A General Theory of Domination and Justice, Oxford.

Lovett, Frank/Pettit, Philip, 2009: Neorepublicanism: A Normative and Institutional Research Program, in: Annual Review of Political Science 12, 11-29.

Marchart, Oliver, 2005: Neu beginnen. Hannah Arendt, die Revolution und die Globalisierung, Wien.

Markell, Patchen, 2008: The Insufficiency of Non-Domination, in: Political Theory 36 (1), 9-36.

Martí, José Luis/Pettit, Philipp, 2010 a: A Political Philosophy in Public Life. Civic Republicanism in Zapatero's Spain, Princeton, NJ.

Martí, José Luis/Pettit, Philip, 2010 b: Giving Philosophy a Public Life, in: dies. 2010 a, 135-160.

Maynor, John, 2003: Republicanism in the Modern World, Farnham.

McCormick, John P., 2006: Contain the Wealthy and Patrol the Magistrates: Restoring Elite Accountability to Popular Government, in: American Political Science Review 100 (2), 147-163.

McCormick, John P., 2007: People and Elites in Republican Constitutions, Traditional and Modern, in: Neil Walker/Martin Loughlin (Hrsg.), The Paradox of Constitutionalism: Constituent Power and Constitutional Form, Oxford, 107-128.

McCormick, John P., 2011: Machiavellian Democracy, Cambridge.

McCormick, John P., 2013: Republicanism and Democracy, in: Andreas Niederberger/Philipp Schink (Hrsg.), Republican Democracy. Liberty, Law and Politics, Edinburgh, 89-128.

McMahon, Christopher, 2005: The Indeterminacy of Republican Policy, in: Philosophy \& Public Affairs 33, 67-93.

Michelman, Frank, 1988: Law's Republic, in: The Yale Law Journal 97 (8), 1493-1537. 
Niederberger, Andreas, 2008: Wieviel Gemeinwohl braucht die Republik? Überlegungen zu neuen Varianten des Republikanismus jenseits von substantieller Gemeinschaft und Tugend, in: J. Böckelmann/F. Meier/C. Morgenroth (Hrsg.), Politik der Gemeinschaft. Zur Konstitution des Subjekts in der politischen Philosophie der Gegenwart, Bielefeld, 87-105.

Niederberger, Andreas, 2009: Republikanismus jenseits der Republik? Zur symbolischen Funktion der Demokratie bei Marcel Gauchet, Claude Lefort, Jacques Rancière und Pierre Rosanvallon, in: M. Hirsch/R. Voigt (Hrsg.), Der Staat in der Postdemokratie. Staat, Politik, Demokratie und Recht im neueren französischen Denken, Stuttgart, 93-113.

Niederberger, Andreas/Schink, Philipp (Hrsg.), 2013: Republican Democracy. Liberty, Law and Politics, Edinburgh.

Pansardi, Pamela, 2013: A Non-Normative Theory of Power and Domination, in: Critical Review of International Social and Political Philosophy 16, 614-633.

Pettit, Philip, 1994: Liberal/Communitarian: MacIntyre's Mesmeric Dichotomy, in: J. Horton/S. Mendus (Hrsg.), After MacIntyre, Oxford.

Pettit, Philip 1999 a: Republicanism: A Theory of Freedom and Government, Oxford.

Pettit, Philip, 1999 b: Republican Freedom and Contestatory Democratization, in:

Casiano Hacker-Cordon/Ian Shapiro (Hrsg.), Democracy's Value, Cambridge, 163-190.

Pettit, Philip, 2000: Democracy: Electoral and Contestatory, in: Ian Shapiro/ Stephen Macedo (Hrsg.), Designing Democratic Institutions, New York, 105-146.

Pettit, Philip, 2001: A Theory of Freedom: From the Psychology to the Politics of Agency, Oxford.

Pettit, Philip, 2004: Depoliticizing Democracy, in: Ratio Juris 17 (1), 52-65.

Pettit, Philip, 2006 a: Two-dimensional Democracy and the International Domain, in: The Monist 89 (2), 301-324.

Pettit, Philip, 2006 b: The Determinacy of Republican Policy. A Reply to McMahon, in: Philosophy \& Public Affairs 34, 275-283.

Pettit, Philip, 2008: Three Conceptions of Democratic Control, in: Constellations 15 (1), 46-55.

Pettit, Philip, 2010: A Republican Law of Peoples in: European Journal of Political Theory 9 (1), 70-94.

Pettit, Philip, 2012: On the People's Terms. A Republican Theory and Model of Democracy, Cambridge. 
Pettit, Philip, 2013: Two Republican Traditions, in: Andreas Niederberger/Philipp Schink (Hrsg.), Republican Democracy. Liberty, Law and Politics, Edinburgh, 169-204.

Pettit, Philip, 2014: Just Freedom. A Moral Compass for a Complex World, New York/London.

Phillips, Anne, 2000: Feminism and Republicanism: Is This a Plausible Alliance?, in: Journal of Political Philosophy 8 (2), 279-293.

Rawls, John, 1979: Eine Theorie der Gerechtigkeit, Frankfurt a. M.

Rosanvallon, Pierre, 2009: Counter Democracy. Politics in an Age of Distrust, Cambridge.

Rosanvallon, Pierre, 2010: Demokratische Legitimität, Hamburg.

Urbinati, Nadia, 2010: Unpolitical Democracy, in: Political Theory 38 (1), 65-92. Sager, Alex, 2014: Political Rights, Republican Freedom, and Temporary Workers, in: Critical Review of International Social and Political Philosophy 17 (2), 189-211.

Schaap, Andrew (Hrsg.), 2009: Law and Agonistic Politics, Farnham.

Schulz, Daniel, 2011: Jenseits von Postdemokratie und Neojakobinismus. Die Demokratiediskussion in der politischen Theorie Frankreichs, in: Zeitschrift für Politikwissenschaft 21 (2); 327-339.

Skinner, Quentin, 1986: The Paradoxes of Political Liberty, in: S. McMurrin (Hrsg.), The Tanner Lectures on Human Values, Bd. 9, Cambridge.

Skinner, Quentin, 1990: The Republican Ideal of Liberty, in: G. Bock/Q. Skinner/ M. Viroli (Hrsg.), Machiavelli and Republicanism, Cambridge, 293-309.

Skinner, Quentin, 1997: Liberty before Liberalism, Cambridge.

Sunstein, Cass R., 1999: One Case at a Time. Judicial Minimalism on the Supreme Court, Cambridge, MA.

Thiel, Thorsten, 2012: Republikanismus und die Europäische Union. Eine Neubestimmung des Diskurses um die Legitimität europäischen Regierens, BadenBaden.

Viroli, Maurizio, 2002: Republicanism, New York.

Volk, Christian, 2013: Zwischen Entpolitisierung und Radikalisierung - Zur Theorie von Demokratie und Politik in Zeiten des Widerstands, in: Politische Vierteljahresschrift 54 (1), 75-110.

Waldron, Jeremy, 2004: Judicial Review and Republican Government, in: C. Wolfe (Hrsg.), That Eminent Tribunal. Judicial Supremacy and the Constitution, Princeton, 159-180.

Waldron, Jeremy, 2006: The Core of the Case Against Judicial Review, in: The Yale Law Review 115, 1346-1406. 
Korrespondenzanschrift:

Dipl.-Pol. Andreas Busen

Heisenberg-Professur „Europa und Moderne“

Institut für Politikwissenschaft

Universität Hamburg

Allende-Platz 1

20146 Hamburg

E-Mail: andreas.busen@wiso.uni-hamburg.de

Dr. Thorsten Thiel

Leibniz-Forschungsverbund „Krisen einer globalisierten Welt“

Hessische Stiftung Friedens- und Konfliktsforschung

Baseler Str. 27-31

60329 Frankfurt am Main

E-Mail: thorsten.thiel@normativeorders.net 